\title{
Influence of Perceived Baseline Contact on the Acceptability of a Personal Contact Intervention Among People Living With Dementia
}

\section{Danielle Sullivan}

University of Alberta

Tynisha Whynot

University of Alberta

Jennifer Swindle

University of Alberta

Heunjung Lee

University of Alberta

Hannah O'Rourke ( $\nabla$ hannah.orourke@ualberta.ca)

University of Alberta

\section{Research Article}

Keywords: Dementia, Loneliness, Interpersonal Relations, Feasibility Studies, Complex Intervention Design

Posted Date: December 28th, 2020

DOI: https://doi.org/10.21203/rs.3.rs-125199/v1

License: @ (i) This work is licensed under a Creative Commons Attribution 4.0 International License. Read Full License 


\section{Abstract}

Background: Living with dementia can be a lonely experience, undermining older adults' quality of life. Evidence supports that personal contact interventions can address older adults' loneliness. However, effects have been small to date, and little is known about how one's perceptions of baseline contact may influence an intervention's use and effects. The purpose of this study was to explore how baseline contact, as perceived by older adults living with dementia, may be associated with the acceptability of a personal contact intervention.

Methods: This descriptive study was a secondary analysis of data that we collected from 15 people living with mild to moderate dementia in a long-term care home who participated in Connecting Today. Connecting Today is a personal contact intervention that involves 30 minutes of scheduled, unscripted interaction between persons living with dementia and a family member, friend or research assistant. Visits occurred for six weeks, face-to-face or by phone. We compared groups that perceived that they had high (i.e., weekly, $n=8$ ) versus low (i.e., monthly or unknown, $n=7$ ) contact at baseline to explore differences in acceptability. We measured perceived acceptability (adapted Treatment Perception and Preferences Questionnaire) and the amount and reasons reported for missing data and study withdrawal. Descriptive statistics were used to summarize the data, and proportions and frequencies were compared in tables and charts to explore differences between groups.

Results: In visits one and two when there were little missing data, a larger proportion (85.7\% to $100 \%)$ of low contact participants reported feeling better, that the visits helped them, and that the visits were easy 'mostly' or 'a lot', as compared to the high contact group (37.5\% to $62.5 \%)$. Retention and attrition after visit two further supported that Connecting Today may be more acceptable for people with low perceived baseline contact: the majority of missing data (71\%) and all study withdrawals were observed in the high contact group.

Conclusions: Testing the hypotheses generated in this study about the influence of perceived baseline contact upon acceptability in larger feasibility and effectiveness studies will support development of tailored, theory-based personal contact interventions.

\section{Background}

Humans are a deeply social species. Social connectedness reflects the feeling of belonging to a group and feeling close to other people [1], and is a valuable resource in life [2]. Conversely, loneliness is associated with poor mental and physical health [3]. Loneliness is also a perception, and reflects feeling that the amount or quality of one's relationships is insufficient to meet one's needs [4-6]. Social isolation is a distinct concept referring to an objective lack of contact with, access to, or interaction with others [7] and is a risk factor for loneliness [1].

Age is negatively related to network size, closeness to network members, and the number of non-primary-group ties, placing older adults at increased risk for social isolation and loneliness [8]. The health risks posed by loneliness may be particularly severe for older adults $[9,10]$, especially when they are also facing stressful life course transitions, health problems such as chronic diseases or psychological stress [11], or disabilities [12]. Dementia is a chronic disease, and there is a steep increase in the prevalence of dementia with ageing: in 2014, an estimated $11.6 \%$ of Canadians between $75-84$ and $37.1 \%$ over age 85 were living with dementia [13].

\section{Personal contact interventions}

Personal contact interventions are among the most frequently used approaches to prevent loneliness among cognitively intact older adults [14]. These interventions are based directly on the empirical finding that meaningful social contact is a key factor that influences feelings of loneliness and social connection among older adults [15-17]. Personal contact interventions are single component interventions [18]: they use scheduled contact with a family member, friend, or volunteer as the component or 'active ingredient' to address loneliness [14]. The promise of personal contact interventions is supported by numerous studies that have found a small but statistically significant reduction in loneliness following a personal contact intervention when used with older adults [19-23].

However, researchers have not evaluated use of personal contact interventions to reduce loneliness specifically among older adults living with dementia. The symptoms associated with dementia include memory problems, behavioural and personality changes, confusion and disorientation [24]. Dementia symptoms increase the risk of feeling lonely due to the decreased ability to initiate and maintain meaningful social connections without appropriate support, and the possibility of forgetting an interaction after it has occurred [8]. Studies of the effects of personal contact interventions that aimed to address loneliness have targeted the 'cognitively 
intact' or people with 'good cognitive function' and have, at most, included a small minority of people who had mild cognitive impairment $[20,22,23]$.

No personal contact intervention aimed to address loneliness has been specifically designed or adapted to meet the needs of people living with mild, moderate or severe dementia [14]. As such, we know little about the appropriateness, potential effectiveness, and necessary adaptations of personal contact interventions for use with people living with dementia. Acceptability refers to key stakeholders' views related to an intervention. Acceptability assessments are done to determine whether an intervention is appropriate, reasonable, suitable and convenient for application in a new target population, future research or in daily life [25]. If key stakeholders, like members of the target population, do not find an intervention acceptable, in terms of its activities, mode of delivery, or dose, then they are unlikely to participate in it, and it cannot have an impact on loneliness.

\section{Collection of Baseline Contact Data}

A key factor that may influence acceptability of a personal contact intervention is the amount and nature of contact that the person living with dementia perceives that they receive at baseline. Along with the limited research attention given to addressing loneliness experienced by older adults living with dementia, few studies of a personal contact intervention have collected data related to the participants' perceptions of their own personal contact at baseline. Most studies have described some social variables at baseline, such as living arrangement, social support, or loneliness [20-23, 26-28], but none considered participants' perceptions of the amount or nature of personal contact prior to implementing a personal contact intervention. This seems to be an important omission, because personal contact interventions aim to increase feelings of connection by augmenting the amount or nature of contact that one receives.

We will describe two examples to illustrate this issue: a qualitative study that examined the opinions of users of the Befriending Scheme, a local home-visiting service, $(n=13)$ [28], and a case study about a program to use email contact $(n=9)$ [19]. Both studies were of personal contact interventions aimed to reduce loneliness among older adults. While each of these studies reported some data related to the participants' baseline contact (e.g., living arrangement, having someone to talk to), neither explored how people's perceptions of their personal contact with others at baseline may have influenced their uptake of, or engagement in, the personal contact intervention. Researchers tend to assume that people who are isolated at baseline (e.g., living alone, or who have few friends) may respond best to interventions aimed at increasing their personal contact, and then design their inclusion criteria accordingly to target the socially isolated $[19,27]$. However, participants with dementia have their own perceptions of the amount and nature of contact that they receive at baseline, and these may differ from more objective measures of social isolation. How these perceptions of baseline contact may influence their opinions of and engagement with a personal contact intervention has not been explored empirically to date.

The purpose of this study was to explore how the perceptions of baseline contact of people living with dementia influenced their acceptability of a personal contact intervention. This was the first study, to our knowledge, to explore these questions. From our findings, we have generated novel hypotheses for testing in future research aimed to advance theory of the mechanisms and effects of personal contact interventions.

\section{Methods}

\section{Aim}

Our aim was to explore how perceptions of weekly (as compared to monthly or unknown) baseline contact may be related to acceptability of "Connecting Today", a personal contact intervention. Connecting Today was pilot tested in a recent study with persons living with mild to moderate dementia in long-term care homes. The overall feasibility and acceptability of Connecting Today will be reported in a forthcoming manuscript.

\section{Design}

This was a quantitative, descriptive, exploratory study, and secondary analysis of data that we collected during the time that participants in either the intervention or wait-list control groups received the Connecting Today intervention. Data were from 15 people living with mild to moderate dementia residing in two urban long-term care homes. All were offered six Connecting Today visits. Ethical 
approval was received from the Health Research Ethics Board - Health Panel at the University of Alberta (Pro00084244). This research was performed in accordance with the Declaration of Helsinki.

\section{The Connecting Today Intervention}

Development of Connecting Today has occurred in phases as per Medical Research Council recommendations for the development of complex interventions[29, 30]. In Phase 1, HMO completed: (i) scoping reviews to define antecedents and outcomes of older adults' loneliness[1], and clarify the intervention's active ingredient, activities, mode of delivery and dose[14]; (ii) a mixed methods study which supported acceptability of personal contact interventions according to health care providers and family members and friends of people living with dementia. This study highlighted the importance of flexible delivery, having a facilitator available to schedule and check-in during visits, to involve family members and friends where feasible, and to bring and set up telephone equipment as needed $(n=25)$ [31]; and (iii) the present study is a secondary analysis of data that we collected as part of Phase 2 pilot testing to assess feasibility and acceptability of Connecting Today.

The essential component of the Connecting Today intervention is providing routine visits for a person living with dementia while they reside in long-term care. We told participants to do what they can to attend and engage in routine visits, and that we would be studying what is actually possible for people living with dementia in long-term care homes and their family members and friends. Some care home residents had someone that self-identified as a family member or friend and was willing to be their personal contact. If they did not have a contact, then the research assistant (RA) was assigned to complete the visits. Several activities were involved to implement routine visits: (1) The person with dementia (or their designated decision maker) chose a family member, friend or the RA to be the contact, and interacted with the same contact person for all visits, face-to-face or by phone. In our study, three residents chose their own visitor (which was the RA in all cases); the other contacts were selected by Designated Decision-Makers (DDMs). Seven of the 15 residents visited with an RA contact. We encouraged visits to occur face-to-face at least half of the time. (2) The RA worked with the friend/friend contact to schedule visits for a minimum of 30 minutes, once per week, for 6 weeks, and sent the personal contact an Alzheimer Society handout related to communication strategies used with people living with dementia. (3) The RA attended each visit as the contact or, in the case of a friend/friend contact, to help set-up for the visit (e.g., find a quiet location, set up the telephone as needed), and to complete a brief check-in after the first 10 minutes. (4) The resident and their contact determined what to talk about or do each visit.

\section{Setting}

The pilot study took place across two large long-term care homes (each $>100$ beds) situated in a mid-size urban setting in Alberta, Canada. The homes were similar in terms of their physical features (e.g., high proportion of shared rooms arranged along corridors) and philosophies of person-centred care.

\section{Inclusion and Exclusion Criteria}

Clinical staff identified potentially eligible participants (items 1-5 below). The RA then confirmed eligibility (item 6). Clinical staff were asked to complete the preliminary eligibility screen in January, April and July 2019 to identify new participants. The eligibility criteria were:

1. Completed Resident Assessment Instrument (RAI) 2.0 full assessment [32] (to assess for eligibility, and necessary for the collection of demographic data).

2. Age of 65 or older (RAl 2.0 item AA3a).

3. Diagnosed with Alzheimer's disease or other dementia (RAI 2.0 items I1r, 11v).

4. Had possible mild-to-moderate cognitive impairment (RAI 2.0 Cognitive Performance Score 1-3) [33].

5. Understood spoken English (based on clinical staff's knowledge of the resident).

6. Had a Mini Mental Status Examination score $\geq 12$ (necessary to complete measures).

\section{Recruitment and Informed Consent}

In consultation with site managers, one or two units within each site were selected that included persons living with mild to moderate dementia. Care home staff reviewed the medical records (i.e., the Resident Assessment Instrument, RAI 2.0 data) of all the residents in 
the selected units to determine eligibility. These staff then approached all residents (or the DDM) that met the inclusion criteria to assess their interest in participating in the study. Clinical staff provided to the RA the names and contact information for those that were interested in learning more about the study. The RA contacted these individuals to explain the study in further detail, confirm eligibility, and obtain written (or audio-recorded verbal) consent from the person living with dementia (or their DDM). The RA also assessed assent of people living with dementia based on verbal and non-verbal indicators during each visit and data collection session. The sample size calculation identified that twenty participants were needed to estimate a proportion of $15 \%$ (which would be a common estimate of attrition, for example) within 9 points of accuracy with $90 \%$ confidence (Hertzog, 2008). To account for withdrawals and missing data, the pilot study investigators aimed to recruit a minimum of 30 participants for the pilot, but challenges with recruitment resulted in a smaller sample size $(n=15)$. The small sample size is a study limitation.

\section{Data Collection}

Data were collected to assess participant demographics, perceptions of baseline contact, and indicators of intervention acceptability from March to November 2019.

Demographics. Demographic data were collected from residents' clinical RAI 2.0 records two weeks before the intervention group received Connecting Today to measure variables that influence loneliness identified in a comprehensive review [1]. The variables were: age (RAI 2.0 item AA3a), sex (AA2), education (AB7), marital status (A5), hearing (C1), vision (D1), functional dependence (Activity of Daily Living Hierarchy Scale items - G1eA, G1hA, G1iA, \& G1jA), depression (Depression Rating Scale items - E1a, E1d, E1f, E1h, E1i, E1I, \& E1m), involvement in facility life (Index of Social Engagement items - F1a, F1b, F1c, F1d, F1e, \& F1f), conflict with others (F2a, F2b, F2c, \& F2d), absence of family member/friend contact (F2e), recent loss of loved one (F2f), family member/significant other involved in care planning (R1b \& R1c), and number of medications (01). Evidence supports the reliability (inter-rater and internal consistency) and validity (criterion, convergent and predictive) of the RAI 2.0 items [34]. Our research team confirmed the level of cognitive impairment by using the Mini Mental Status Examination (MMSE) [35]. The MMSE is widely used, and has evidence supporting its construct validity and test-retest and interrater reliability [35].

Baseline contact data. The perceptions of the amount and nature of current visits were collected as a component of the demographic information from participants living with dementia before receipt of Connecting Today (Additional file 1). Items were generated by the authors to explore whether Connecting Today offered something different from what the resident perceived that they already received (they were not intended to objectively measure actual contact). The stems were written in simple language, a limited set of response options were provided, and the response tree strategy was applied to enhance comprehension and limit cognitive burden [36]. The RA read each question and the response options aloud in face-to-face sessions. Participants were asked about who visits other than health care workers, and asked about specific types of visitors one at a time (participant responded yes or no). If a participant stated 'yes' to the type of visitor, they were asked how the visits with that person usually happen (in person, over the phone, videoconference, or other). Another item asked, in general, 'how often do people visit' (less than once per month, about once per month, about once per week, a few times per week, every day), and whether visits last as long as the person would like (yes or no). The co-author team, which includes gerontological researchers and people who have experience working directly with people living with dementia, reviewed the items and agreed that they were clear and relevant to the topic under study (face validity).

Acceptability data. Immediately following each Connecting Today visit, the RA asked the resident six items face-to-face to directly measure acceptability, defined as participants' perceptions of appropriateness, effectiveness, and convenience of Connecting Today (Additional file 2). The items were informed by the Treatment Perception and Preference (TPP) measure, but adapted to fit with the particular treatment under assessment (i.e., Connecting Today), as per the developer guidelines $(a>.85)$ [37]. To reduce cognitive burden, item stems were simplified and response options reduced from a 5-point to a 3-point Likert scale, and presented in a responsetree format [36]. Items asked about whether they enjoyed the visit, felt better after the visit, and found it easy to visit. If the participant responded 'yes' to the item, then the level of enjoyment, feeling better, or ease was assessed (a little, mostly, a lot).

Throughout the study, we used a study log to collect data related to retention, attrition, attendance at visits, and reasons that persons living with dementia or their DDMs gave for not consenting to participate in the study. These are indirect indicators of acceptability because withdrawal, non-attendance, and non-consent are associated with perceptions of the intervention; people who find an intervention unacceptable are less likely to agree to participate or to complete all intervention sessions [38]. 


\section{Data Analysis}

Descriptive statistics (means/SDs or medians and interquartile ranges for continuous variables and frequencies, medians, and modes for categorical variables) were used to summarize the demographic characteristics and perceptions of baseline contact. We dichotomized the variable measuring visit frequency to define two subgroups: (1) persons living with dementia who received weekly visits prior to the intervention and (2) persons living with dementia who received monthly visits or did not know their baseline contact prior to the intervention. Descriptive statistics were used to summarize acceptability data in the overall sample (means/SDs as sample size was 15) and the two subgroups (frequencies as the sample sizes were smaller). For analysis of attendance, attrition and missing data, the denominator was the total number in the subgroup at baseline. The proportion of missing data at each time point and the reasons for missing data were analyzed and considered carefully in the interpretation of the results. For acceptability data, we used complete case analysis. Conventional content analysis [39] was used to summarize the reasons for non-consent. These data were then converted to a quantitative, categorical format based on the identified reasons for non-consent. We used tables and bar charts to compare (not test) the results between the two subgroups for each visit (six visits in total). Between the groups, we noted differences in perceived acceptability, and withdrawal and non-attendance. We explored whether the reasons for non-consent were related to baseline contact. This led us to generate hypotheses about the influence of baseline contact on acceptability.

\section{Results}

\section{Recruitment and non-consent}

The staff at both sites approached all 103 eligible residents or their DDM and provided information about the study. Of those residents/ DDMs, 69 showed interest. When approached by the RA, 35 persons living with dementia or their DDMs declined to participate and 13 did not meet the inclusion criterion of having an MMSE $\geq 12$, leaving a total of 15 participants. The RA recorded the reasons that people living with dementia/ DDMs decided not to participate in the study. The most common reasons for non-consent were perceptions that the intervention or study was not appropriate for the health condition or ability of the person living with dementia $(n=16)$ and concerns with asking the person living with dementia too many questions $(n=9)$. Several people also declined to participate because they perceived that the person living with dementia already received enough visits $(n=5)$ (see Figure 1$)$.

\section{Sample characteristics}

The sample was mainly female $(n=13,86.7 \%)$, the average age was 87.2 years (range=76-99) and a majority of participants were widowed $(n=9,60.0 \%)$. This sample composition is typical of care home populations in Canada. Nine out of 15 persons living with dementia (60.0\%) had hearing impairment and six (40.0\%) had vision impairment. The average level of functional dependence was 3 , indicating needs of extensive assistance in personal hygiene or toilet use score (scale range 0-6; 0=independent 6-total dependence). The average depression score was 2.13 (SD 2.61); a score of 3 or more indicates a potential or actual problem with depression (scale range $0-14$ ). None of the persons living with dementia had total absence of family member/friend contact or had recently lost a loved one. Most residents did not have a family member or significant others $(n=9,60 \%)$ involved in the RAl 2.0 assessment care planning (see Table 1). We did not observe any obvious differences in the demographic characteristics between the groups with weekly as compared to monthly or unknown contact at baseline.

\section{Perceived baseline contact}

How often a participant perceived they had a visitor was divided almost equally between groups that perceived high contact (i.e., having visitors at least once per week, $n=8$ ), and low/ unknown contact (i.e., having visitors once per month or not remembering the frequency of contact, $n=7)$. Participants most often reported that visitors were their children $(n=9,60.0 \%)$ or a friend $(n=8,53.3 \%)$. These visits occurred most often in-person (children 7 of $9,77.8 \%$; friend 5 of $8,62.5 \%$ ) (see Table 2).

\section{Attendance, attrition, and missing data}

Participant attendance at sessions differed between the high and low baseline contact groups (see Table 3). In the high baseline contact group, four withdrawals occurred between visits two to six and contributed to the higher non-attendance rates observed in this group. No participants from the low baseline contact subgroup withdrew from the study. Two of the four withdrawals were related to perceptions of the study or intervention. The participant who withdrew after visit one stated, 'I don't think this will help'. She explained she was too busy in the mornings for the visits and too tired in the afternoons, indicators that the intervention was not viewed as 
appropriate by this participant. Another participant was withdrawn by their DDM after visit three because their DDM reported that they were agitated and annoyed by the questions the RA asked. This suggests that the intervention was not viewed positively enough to outweigh the inconvenience of the study procedures.

There were 9 instances of missing data, and 7 (71\%) of these instances occurred in the high baseline contact group. Some missing data in both groups could be explained by reasons that did not have to do with Connecting Today or study procedures. These reasons included the RA being unable to attend visit three (affected $n=1$ in the high contact and $n=2$ in the low contact group) and an influenza outbreak at the site during visit six (affected $n=2$ in each group). The other instances of missing data occurred because the participant or their family member/friend contact did not attend the session or the participant refused to answer the questions. These instances of missing data could be related to perceptions of Connecting Today or study procedures and occurred most often in the high contact group $(n=4)$ as compared to the low contact group $(n=1)$. Taken together, the patterns related to missing data and attrition suggest that participants with a lower perceived baseline contact may stay in the study longer and participate more regularly than participants with a higher perceived baseline contact.

\section{Perceived acceptability}

In visits 1 and 2, there were little missing data, and the majority of the 15 participants that attended sessions perceived Connecting Today as enjoyable, effective to help them feel better, and that it was easy to visit with the contact (i.e., majority rating $\geq 2$ for each item). However, there were some differences between high and low contact groups in the proportion rating Connecting today as enjoyable, effective, and easy (i.e., $\geq 2$ ) during visits 1 and 2 that suggested that the low contact group may have held more positive perceptions of Connecting Today. Missing data in visits 4 to 6 limited our ability to draw conclusions about the extent to which these perceptions were maintained over time (see Table 3). These findings are presented in detail below.

\section{Appropriateness.}

During visits 1 and 2, the majority of participants enjoyed the visits in both the low (100\%) and high contact groups (87.5\%). However, in visits 1 and 2, a higher proportion of people in the low contact group enjoyed visits "mostly" or "a lot" (100\%), as compared to the high contact group (62.5\%). In visits 4, 5, and 6, there were several participants in the low contact group that reported enjoying visits "a lot" $(n=4,6,1)$. In contrast, in the high baseline contact group in visits 4,5 and 6 , very few reported that they enjoyed the visit "a lot" $(n=2,1,1)$.

\section{Effectiveness.}

Effectiveness (as a component of acceptability) refers to the participants' perceptions of the intervention in terms of helpfulness in managing present problems [25]. In visits 1 and 2, the majority of participants reported that Connecting Today helped them feel better in both the low (85.7\%) and high contact groups (75\%). In visits 1 and 2, a higher proportion of people in the low contact group stated that visits helped them feel better mostly or a lot (85.7\%), as compared to the high contact group (37.5\% in visit 1 and $50.0 \%$ in visit 2 ). In visits 4, 5, and 6, there were several people in the low contact group that reported that visits helped them feel a lot better ( $\mathrm{n}=4,5,2)$. In contrast, in the high baseline contact group, very few reported that visits 4,5 , and 6 helped them feel a lot better $(n=1,1,0)$. These patterns support that participants who have a lower perceived baseline contact may view the intervention as more helpful or effective.

\section{Convenience.}

The third question was about the ease of the visit from the perspective of the participants, which relates to convenience. In visits 1 and 2 , all participants in the low contact group (100\%) and about half in the high contact group (50\% in visit 1 and $62.5 \%$ in visit 2 ) found it easy to visit. In visits 1 and 2, a higher proportion of people in the low contact group reported the ease of the visit as 'mostly' or 'a lot' (100.0\% in visit 1 and $85.7 \%$ in visit 2$)$, as compared to the high contact group (50.0\%-62.5\%). In visits 4, 5, and 6, there were several people in the low contact group that stated that ease of visit was 'a lot' $(n=5,6,1)$. In contrast, in the high baseline contact group, very few reported that the ease of visit was 'a lot' in visits 4,5 , and $6(n=2,1,0)$.

\section{Discussion}

In this study, we explored several indicators of acceptability of a personal contact intervention, Connecting Today, in a small sample of people living with mild to moderate dementia in care homes. Considering both attendance and perceived acceptability, we found that a majority of those with high and low baseline contact initially reported that Connecting Today was acceptable. However, after visit three,

Page $7 / 21$ 
patterns of and the reasons for attrition and missing data support that those with high contact at baseline did not find Connecting Today as valuable as those in the low baseline contact group. This proposition was also supported by the consistent pattern of higher proportions of people in the lower contact group reporting that Connecting Today was enjoyable, helped them feel better, and was easy 'mostly' or 'a lot'. Our findings suggest that people living with dementia who perceive their baseline contact to be substantially less than what is offered by a proposed personal contact intervention may have more positive perceptions of the intervention.

The three main hypotheses generated from this small exploratory study, and which should be tested in future research are: (1) Those with high perceived baseline contact are more likely to withdraw from the study or refuse to answer study questions, as compared to those with low contact at baseline; (2) Those with low perceived baseline contact view personal contact interventions as more acceptable as compared to those with high perceived baseline contact; and (3) While both those with low and high perceived baseline contact may benefit from personal contact interventions, effects may be stronger among those with low perceived baseline contact. These hypotheses are novel; the influence of perceived baseline contact on acceptability and subsequent intervention effectiveness has not been previously explored among cognitively intact older adults or people living with dementia.

Our findings support that the collection of perceived baseline contact data may help identify those who would benefit most from a personal contact intervention. Targeting is a commonly used technique that focuses on the development of a single specialized health intervention approach for a defined subgroup of people [40]. Our findings highlight that not all people living with dementia in a longterm care home perceive that they are socially isolated, and that the strongest effects of a personal contact intervention may be observed by targeting those that perceive that they are socially isolated. However, we do not recommend excluding people with high perceived baseline contact from all future studies. While we identified possible differences between the groups highlighting the importance of assessing the participants' perceptions of their baseline contact, some participants in the high baseline contact group still perceived Connecting Today as enjoyable and beneficial and saw value in an intervention that promoted weekly visits.

Rather than excluding people with high perceived baseline contact from future studies, we recommend completing subgroup analyses to compare effects between groups. This approach would advance knowledge because previous studies have overlooked the potential influence of perceived baseline contact or only included people that the researcher deemed socially isolated (e.g., older people living alone), without assessing the individual's perceptions of their social integration [20-23, 26-28]. Participants' perspectives can help researchers gain insight into what works, what does not work, and why. This kind of understanding supports implementation of interventions at the right time to the right person, enhancing their effects, and addressing the urgent need for precise, theory-informed interventions to address individual's needs [41].

Given our findings, Connecting Today will be revised to more explicitly encourage the resident or DDM to tailor the intervention to the resident's needs. Most complex interventions do involve some level of tailoring as they are implemented [42]. Intervention tailoring reflects a process of individual assessment to determine the best strategies for that person based on their characteristics and to address the outcome of interest [40]. For example, some persons living with dementia may perceive that they receive regular visits from a single contact or caregiver, and Connecting Today could be applied to promote visits by people other than the primary contact. The intervention could also be used to offer visits by phone for someone who cannot use the phone on their own. Tailoring would involve a collaborative discussion to identify how Connecting Today could be used to fit the individual's needs and wants for social connection. By considering participants' interests, goals, and perspectives, there is an opportunity to improve adherence, optimize intervention design, and promote better outcomes [25].

Our findings also support that participants' perspectives should be taken into account in future research to design and understand the effects of personal contact interventions. In previous studies with the cognitively intact, participant perspectives have been used to inform the design of an intervention by addressing older adults' concerns related to use of technology in a videoconference intervention [20], to use older adults' suggestions to publicize a telephone befriending service [27], to help select the contact for a befriending program [28], and to identify reasons (e.g., technical problems) why users were satisfied or dissatisfied with a telehealth intervention [23]. These four studies used perspectives of cognitively intact older adults related to the intervention to improve adherence, optimize intervention design, and to promote better outcomes. Our findings support that older adults with dementia also have an important perspective that can be used to inform intervention design, and that their perceived baseline contact may be a key component of this.

\section{Strengths And Limitations}


This was a secondary analysis of a small dataset, and the sub-groups in our study were small. While the small sample greatly limits stability of the quantitative estimates and the findings do not have statistical generalizability, our purpose in this secondary analysis was to use the data to think more deeply about acceptability and generate hypotheses for future testing. The findings support recommendations for improving recruitment and retention in future studies and raise testable questions, and thus have analytic generalizability [43]. Future prospective studies, powered to detect differences between low and high contact groups, will be needed to rigorously test the hypotheses and produce findings with statistical generalizability. After visit three, attrition and missing data lead to very small and unequal group sizes, and comparisons of perceived acceptability between groups after visit two was particularly problematic. Despite missing data, the longitudinal dataset remained a key study strength and supported triangulation of data sources, allowing us to compare perceived acceptability with the amount and reasons for missing data and withdrawals in order to generate the hypotheses.

\section{Conclusion}

This study supports the growing body of literature related to the importance of understanding and integrating perspectives of end users, including people living with dementia, during the research process. Little is known about how perceived baseline contact impacts one's experiences in receiving an intervention aimed at increasing personal contact, and even less is known when the intervention is aimed to support people living with dementia. Our findings support that a person with dementia's perceptions of their baseline contact may affect acceptability of a personal contact intervention. This effect on acceptability will, in turn, influence the potential of such interventions to promote social connectedness and quality of life. This exploratory study has identified patterns that can inform design of future research and has generated novel hypotheses that warrant further testing. Perhaps most important, the findings add support to a growing body of research and advocacy efforts to include the voice of the person with dementia in research. Regardless of whether or not these perceptions hold objective accuracy, the perceptions of people living with dementia related to their baseline contact hold great value to improve understanding of how interventions can be designed to more effectively address loneliness.

\section{List Of Abbreviations}

DDM: designated decision-maker

MMSE: Mini Mental Status Examination

RA: research assistant

RAI: Resident Assessment Instrument

TPP: Treatment Perception and Preference

\section{Declarations}

Ethics approval and consent to participate: Written or oral consent for participation was obtained. Assent to participate was assessed before and during every research activity. Ethical approval was received from the Health Research Ethics Board-Health Panel at the University of Alberta (Pro00084244).

Consent for publication: Not applicable.

Availability of data and materials: The datasets generated and/or analysed during the current study are not publicly available because participants consented to share data only with the research team. De-identified data are available from the corresponding author on reasonable request.

Competing interests: The authors declare that they have no competing interests.

Funding: This work was supported by an Alzheimer Society New Investigator Grant (19-21) and a Faculty of Nursing, University of Alberta Professorship in Dementia Care Interventions, and a University of Alberta summer studentship (awarded to DS). The funders were not involved in the study design, or the collection, analysis or interpretation of data. 
Authors' contributions: DS and TDW contributed equally to this manuscript. They supported conceptualization of research questions for this secondary analysis, completed data analysis and interpretation, and wrote a first draft of the manuscript. JS supported pilot study design, prepared ethics applications and oversaw all data collection. HL participated in data collection and completed data analysis. HMO designed and obtained funding for the pilot study, conceptualized the research questions for this secondary analysis, oversaw analysis, completed interpretation of findings, and wrote and made substantial revision to manuscript drafts. All authors read and approved the final manuscript.

Acknowledgements: We thank Kelti Barsan and Madeleine Pagnucco-Renaud for collecting pilot study data used for this secondary analysis.

\section{References}

1. O'Rourke H, Sidani S. Definition, determinants, and outcomes of social connectedness for older adults: a scoping review. J Gerontol Nurs. 2017;43:43-52.

2. Lee R, Draper M, Lee S. Social connectedness, dysfunctional interpersonal behaviors, and psychological distress: testing a mediator model. J Couns Psychol. 2001;48:310-8.

3. Holt-Lunstad J, Smith TB, Baker M, Harris T, Stephenson D. Loneliness and social isolation as risk factors for mortality: a metaanalytic review. Perspect Psychol Sci. 2015;10:227-37. doi:10.1177/1745691614568352.

4. de Jong Gierveld J. A review of loneliness: concept and definitions, determinants and consequences. Rev Clin Gerontol. 1998;8:7380.

5. El Sadr CB, Noureddine S, Kelley J. Concept analysis of loneliness with implications for nursing diagnosis. Int J Nurs Terminol Classif. 2009;20:25-33. doi:10.1111/j.1744-618X.2008.01110.x.

6. Weiss RS. Loneliness: The experience of emotional and social isolation. Cambridge, MA: MIT Press; 1973.

7. Ashida S, Heaney CA. Differential associations of social support and social connectedness with structural features of social networks and the health status of older adults. J Aging Health. 2008;20:872-93.

8. Cornwell EY, Waite LJ. Social disconnectedness, perceived isolation, and health among older adults. J Health Soc Behav. 2009;50:31-48.

9. Cacioppo JT, Hawkley LC. Social isolation and health, with an emphasis on underlying mechanisms. Perspect Biol Med. 2003;46 SUPPL 3:S39-52.

10. Tomaka J, Thompson S, Palacios R. The relation of social isolation, loneliness, and social support to disease outcomes among the elderly. J Aging Health. 2006;18:359-84.

11. Richard A, Rohrmann S, Vandeleur CL, Schmid M, Barth J, Eichholzer M. Loneliness is adversely associated with physical and mental health and lifestyle factors: results from a Swiss national survey. PLoS One. 2017;12:1-19.

12. Brummett BH, Barefoot JC, Siegler IC, Clapp-Channing NE, Lytle BL, Bosworth HB, et al. Characteristics of socially isolated patients with coronary artery disease who are at elevated risk for mortality. Psychosom Med. 2001;63:267-72.

13. Alzheimer Society of Canada. Prevalence and monetary costs of dementia in Canada: a report by the Alzheimer Society of Canada. 2016. https://alzheimer.ca/sites/default/files/files/national/statistics/prevalenceandcostsofdementia_en.pdf. Accessed 7 Oct 2020.

14. O'Rourke H, Collins L, Sidani S. Interventions to address social connectedness and loneliness for older adults: a scoping review. BMC Geriatr. 2018;18:214.

15. Moyle W, Kellett U, Ballantyne A, Gracia N. Dementia and loneliness: an Australian perspective. J Clin Nurs. 2011;20:1445-53. doi:10.1111/j.1365-2702.2010.03549.x.

16. Cattan M, Newell C, Bond J, White M. Alleviating social isolation and loneliness among older people. Int J Ment Health Promot. 2003;5:20-30. doi:10.1080/14623730.2003.9721909.

17. Pettigrew S, Roberts M. Addressing loneliness in later life. Aging Ment Health. 2008;12:302-9. doi:10.1080/13607860802121084.

18. Sidani S. Health intervention research: Understanding research design and methods. Thousand Oaks, CA: Sage Publications Ltd; 2015.

19. Moses B. Technology as a means of reducing loneliness in the elderly [dissertation]. Ann Arbor, IL: Walden University; 2003. 
20. Schwindenhammer TM. Videoconferencing intervention for depressive symptoms and loneliness in nursing home elders [dissertation]. Ann Arbor, IL: Illinois State University; 2014.

21. Tsai H-H, Tsai Y-F, Wang H-H, Chang Y-C, Chu HH. Videoconference program enhances social support, loneliness, and depressive status of elderly nursing home residents. Aging Ment Health. 2010;14:947-54. doi:10.1080/13607863.2010.501057.

22. Tsai H-H, Tsai Y-F. Changes in depressive symptoms, social support, and loneliness over 1 year after a minimum 3-month videoconference program for older nursing home residents. J Med Internet Res. 2011;13:e93. doi:10.2196/jmir.1678.

23. van der Heide LA, Willems CG, Spreeuwenberg MD, Rietman J, de Witte LP. Implementation of CareTV in care for the elderly: The effects on feelings of loneliness and safety and future challenges. Technol Disabil. 2012;24:283-91. doi:10.3233/TAD-120359.

24. Alzheimer Society of Canada. What is dementia? 2020. https://alzheimer.ca/en/about-dementia/what-dementia. Accessed 29 Oct 2020.

25. Sidani S, Braden CJ. Design, evaluation, and translation of nursing interventions. West Sussex, UK: John Wiley \& Sons, Inc.; 2011.

26. Van Orden KA, Stone DM, Rowe J, McIntosh WL, Podgorski C, Conwell Y. The Senior Connection: design and rationale of a randomized trial of peer companionship to reduce suicide risk in later life. Contemp Clin Trials. 2013;35:117-26. doi:10.1016/j.cct.2013.03.003.

27. Cattan M, Kime N, Bagnall AM. The use of telephone befriending in low level support for socially isolated older people - an evaluation. Heal Soc Care Community. 2011;19:198-206.

28. Andrews GJ, Gavin N, Begley S, Brodie D. Assisting friendships, combating loneliness: users views on a befriending scheme. Ageing Soc. 2003;23:349-62.

29. Campbell M, Fitzpatrick R, Haines A, Kinmonth AL, Sandercock P, Spiegelhalter D, et al. Framework for design and evaluation of complex interventions to improve health. BMJ. 2000;321:694-6.

30. Campbell NC, Murray E, Darbyshire J, Emery J, Farmer A, Griffiths F, et al. Designing and evaluating complex interventions to improve health care. BMJ. 2007;334:455-9.

31. O'Rourke HM, Sidani S, Jeffery N, Prestwich J, McLean H. Acceptability of personal contact interventions to address loneliness for people with dementia: An exploratory mixed methods study. Int J Nurs Stud Adv. 2020;2 September:100009. doi:10.1016/j.jinsa.2020.100009.

32. Morris JN, Hawes C, Mor V, Phillips C, Fries BE, Nonemaker S, et al. Resident Assessment Instrument (RAI) MDS 2.0 User's Manual, Canadian Version. Washington DC: interRAl; 2010.

33. Morris JN, Fries BE, Mehr DR, Hawes C, Phillips C, Mor V, et al. MDS Cognitive Performance Scale. J Gerontol. 1994;49:M174-82.

34. Poss JW, Jutan NM, Hirdes JP, Fries BE, Morris JN, Teare GF, et al. A review of evidence on the reliability and validity of Minimum Data Set data. Healthc Manage Forum. 2008;21:33-9.

35. Folstein MF, Folstein SE, McHugh PR. "Mini-mental state". A practical method for grading the cognitive state of patients for the clinician. J Psychiatr Res. 1975;12:189-98.

36. Fox MT, Sidani S, Streiner DL, McGilton KS, Grady C. Effect of the response tree format on the utility and psychometric properties of scales measuring symptoms in adults undergoing inpatient rehabilitation. Res Nurs Health. 2011;34:508-19.

37. Sidani S, Epstein D, Fox M, Miranda J. Psychometric properties of the Treatment Perception and Preferences measure. Clin Nurs Res. 2018;27:743-61.

38. Sekhon M, Cartwright M, Francis J. Acceptability of healthcare interventions: an overview of reviews and development of a theoretical framework. BMC Health Serv Res. 2017;17:1-13. doi:10.1186/s12913-017-2031-8.

39. Hsieh H, Shannon SE. Three approaches to qualitative content analysis. Qual Health Res. 2005;15:1277-88.

40. Kreuter M, Wray R. Tailored and targeted health communication: strategies for enhancing information relevance. Am J Health Behav. 2003;27 Suppl 3:S227-32.

41. Akhter-Khan SC, Au R. Why loneliness interventions are unsuccessful: a call for precision health. Adv Geriatr Med Res. 2020;2:e200016. doi:10.20900/agmr20200016

42. Moore GF, Audrey S, Barker M, Bond L, Bonell C, Hardeman W, et al. Process evaluation of complex interventions: Medical Research Council guidance. BMJ. 2015;350:1-7.

43. Polit DF, Beck CT. Generalization in quantitative and qualitative research: myths and strategies. Int J Nurs Stud. 2010;47:1451-8. 


\section{Tables}

Table 1 - Resident Demographics ( $n=15)$

Page 12/21 


\begin{tabular}{|c|c|}
\hline Variable & $\mathbf{N}(\%)$ \\
\hline \multicolumn{2}{|l|}{ Sex } \\
\hline Male & $2(13.3)$ \\
\hline Female & $13(86.7)$ \\
\hline Not reported & $0(0.0)$ \\
\hline \multicolumn{2}{|l|}{ Education } \\
\hline No schooling & $0(0.0)$ \\
\hline Grades $1-8$ or less & $1(6.7)$ \\
\hline 9-11 grades & $2(13.3)$ \\
\hline High school graduate & $3(20.0)$ \\
\hline Technical/trade school & $0(0.0)$ \\
\hline Some college & $0(0.0)$ \\
\hline Bachelor's degree & $1(6.7)$ \\
\hline Graduate degree & $0(0.0)$ \\
\hline \multirow[t]{2}{*}{ Not reported } & $8(53.3)$ \\
\hline & Not assessed at 1 site \\
\hline \multicolumn{2}{|l|}{ Marital Status } \\
\hline Never married & $1(6.7)$ \\
\hline Married & $2(13.3)$ \\
\hline Widowed & $9(60.0)$ \\
\hline Separated & $0(0.0)$ \\
\hline Divorced & $3(20.0)$ \\
\hline Not reported & $0(0.0)$ \\
\hline \multicolumn{2}{|l|}{ Hearing } \\
\hline Hears adequately & $5(33.3)$ \\
\hline Minimal difficulty & $4(26.7)$ \\
\hline Hears in special situations only & $2(13.3)$ \\
\hline Highly impaired/absence of useful hearing & $0(0.0)$ \\
\hline Not reported & $4(26.7)$ \\
\hline \multicolumn{2}{|l|}{ Vision } \\
\hline Adequate & $2(13.3)$ \\
\hline Impaired & $6(40.0)$ \\
\hline Moderately impaired & $1(6.7)$ \\
\hline Highly impaired & $2(13.3)$ \\
\hline Severely impaired & $0(0.0)$ \\
\hline
\end{tabular}




\begin{tabular}{|c|c|}
\hline Variable & $\mathbf{N}(\%)$ \\
\hline Not reported & $4(26.7)$ \\
\hline \multicolumn{2}{|c|}{ Functional dependence } \\
\hline Independent & $0(0.0)$ \\
\hline Supervision & $0(0.0)$ \\
\hline Limited & $5(33.3)$ \\
\hline Extensive 1 & $4(26.7)$ \\
\hline Extensive 2 & $6(40.0)$ \\
\hline Dependent & $0(0.0)$ \\
\hline Total dependence & $0(0.0)$ \\
\hline Not reported & $0(0.0)$ \\
\hline \multicolumn{2}{|l|}{ Conflict } \\
\hline \multicolumn{2}{|l|}{ With staff } \\
\hline No & $14(93.3)$ \\
\hline Yes & $1(6.7)$ \\
\hline Not reported & $0(0.0)$ \\
\hline \multicolumn{2}{|l|}{ With roommate } \\
\hline No & $15(100.0)$ \\
\hline Yes & $0(0.0)$ \\
\hline Not reported & $0(0.0)$ \\
\hline \multicolumn{2}{|l|}{ Other residents } \\
\hline No & $14(93.3)$ \\
\hline Yes & $1(6.7)$ \\
\hline Not reported & $0(0.0)$ \\
\hline \multicolumn{2}{|c|}{ Family member/friends } \\
\hline No & $15(100.0)$ \\
\hline Yes & $0(0.0)$ \\
\hline Not reported & $0(0.0)$ \\
\hline \multicolumn{2}{|c|}{ Absence of family member/friend contact } \\
\hline No & $15(100.0)$ \\
\hline Yes & $0(0.0)$ \\
\hline Not reported & $0(0.0)$ \\
\hline \multicolumn{2}{|c|}{ Recent loss of a loved one } \\
\hline No & $15(100.0)$ \\
\hline Yes & $0(0.0)$ \\
\hline
\end{tabular}




\begin{tabular}{|ll|}
\hline Variable & $\mathbf{N}(\%)$ \\
\hline \multicolumn{1}{|c|}{ Not reported } & \\
\hline Family member/significant other involved in RAI 2.0 assessment & \\
\hline No & $9(0.0)$ \\
\hline Yes & $6(40.0)$ \\
\hline Not reported & $0(0.0)$ \\
\hline
\end{tabular}

Table 2 -Perceived baseline contact reported by persons living with dementia ( $n=15)$ 


\section{Anyone visits}

$\begin{array}{ll}\text { No } & 2(13.3) \\ \text { Yes } & 13(86.7)\end{array}$

\section{Who visits}

Friends

Spouse

Children

Grandchildren

Siblings

A paid companion

Volunteer(s)

Cousin(s)

Nieces or nephews

Other
$8(53.3)$

$3(20.0)$

$9(60.0)$

$6(40.0)$

$6(40.0)$

$0(0.0)$

$6(40.0)$

2 (13.3)

4 (26.7)

3 (20.0) [Neighbors, Pastor, Don't know where she came from]

\section{Typically in-person visits}

Friends $(\mathrm{n}=8)$

Spouse $(n=3)$

Children $(n=9)$

Grandchildren $(n=6)$

Siblings $(n=6)$

A paid companion $(n=0)$

Volunteer(s) $(n=6)$

Cousin(s) $(n=2)$

Nieces or nephews $(n=4)$

Other $(n=3)$

\section{Typically phone visits}

\begin{tabular}{ll} 
Friends $(\mathrm{n}=8)$ & $2(25.0)$ \\
Spouse $(\mathrm{n}=3)$ & $1(33.3)$ \\
\hline Children $(\mathrm{n}=9)$ & $2(22.2)$ \\
Grandchildren $(\mathrm{n}=6)$ & $1(16.7)$ \\
Siblings $(\mathrm{n}=6)$ & $2(33.3)$ \\
A paid companion $(\mathrm{n}=0)$ & $0(0.0)$ \\
Volunteer $(\mathrm{s})(\mathrm{n}=6)$ & $1(16.7)$ \\
Cousin $(\mathrm{s})(\mathrm{n}=2)$ & $1(50.0)$
\end{tabular}

$5(62.5)$

$2(66.7)$

$7(77.8)$

$5(83.3)$

$4(66.7)$

$0(0.0)$

5 (83.3)

1 (50.0)

3 (75.0)

3 (100.0)

$1(50.0)$ 
Nieces or nephews $(n=4)$

$1(25.0)$

Other $(n=3)$

$0(0.0)$

\section{Typically visits both in-person and by phone}

$\begin{array}{ll}\text { Friends }(n=8) & 0(0.0) \\ \text { Spouse }(n=3) & 0(0.0) \\ \text { Children }(n=9) & 0(0.0) \\ \text { Grandchildren }(n=6) & 0(0.0) \\ \text { Siblings }(n=6) & 0(0.0) \\ \text { A paid companion }(n=0) & 0(0.0) \\ \text { Volunteer }(s)(n=6) & 1(16.7) \\ \text { Cousin }(s)(n=2) & 1(50.0) \\ \text { Nieces or nephews }(n=4) & 1(25.0) \\ \text { Other }(n=3) & 0(0.0)\end{array}$

\section{Number of visitor types}

\begin{tabular}{ll}
0 visitors & $2(13.3)$ \\
1 visitors & $0(0.0)$ \\
\hline 2 visitors & $3(20.0)$ \\
\hline 3 visitors & $4(26.7)$ \\
4 visitors & $3(20.0)$ \\
5 visitors & $1(6.7)$ \\
6 visitors & $2(13.3)$
\end{tabular}

\section{Frequency of contact}

\begin{tabular}{ll} 
Everyday & $0(0.0)$ \\
\hline A few times per week, but not everyday & $3(20.0)$ \\
\hline Once per week & $5(33.3)$ \\
\hline About once per month & $4(26.7)$ \\
\hline Less than once per month & $0(0.0)$ \\
\hline Don't know & $3(20.0)$ \\
Not reported & $0(0.0)$
\end{tabular}

\section{Length of contact/visit (in minutes)}

\begin{tabular}{ll} 
Less than 60 & $3(20.0)$ \\
60 to 120 & $5(33.3)$ \\
\hline More than 120 & $1(6.7)$ \\
\hline Didn't report in minutes & $5(33.3)$ [Depends on the day; Doesn't matter; Good enough; Not sure; Hard to \\
& say] \\
Not reported & $1(6.7)$
\end{tabular}




\begin{tabular}{|ll|}
\hline Variable & $\mathbf{N}(\%)$ \\
\hline $\begin{array}{l}\text { Acceptability of length of visit (resident } \\
\text { perception) }\end{array}$ \\
\hline No & $3(20.0)$ \\
\hline Yes & $9(60.0)$ \\
\hline Don't know & $2(13.3)$ \\
\hline Not reported & $1(6.7)$ \\
\hline Identify with a culture or ethnicity & \\
\hline No & $12(80.0)$ \\
\hline Yes (Canadian [n=1]; Ukrainian Catholic [n=1]) & $2(13.3)$ \\
\hline Don't know & $1(6.7)$ \\
\hline Not reported & $0(0.0)$ \\
\hline
\end{tabular}

Table 3. Perceived acceptability in high (weekly, $n=8$ ) or low (monthly or unknown, $n=7$ ) baseline contact groups 


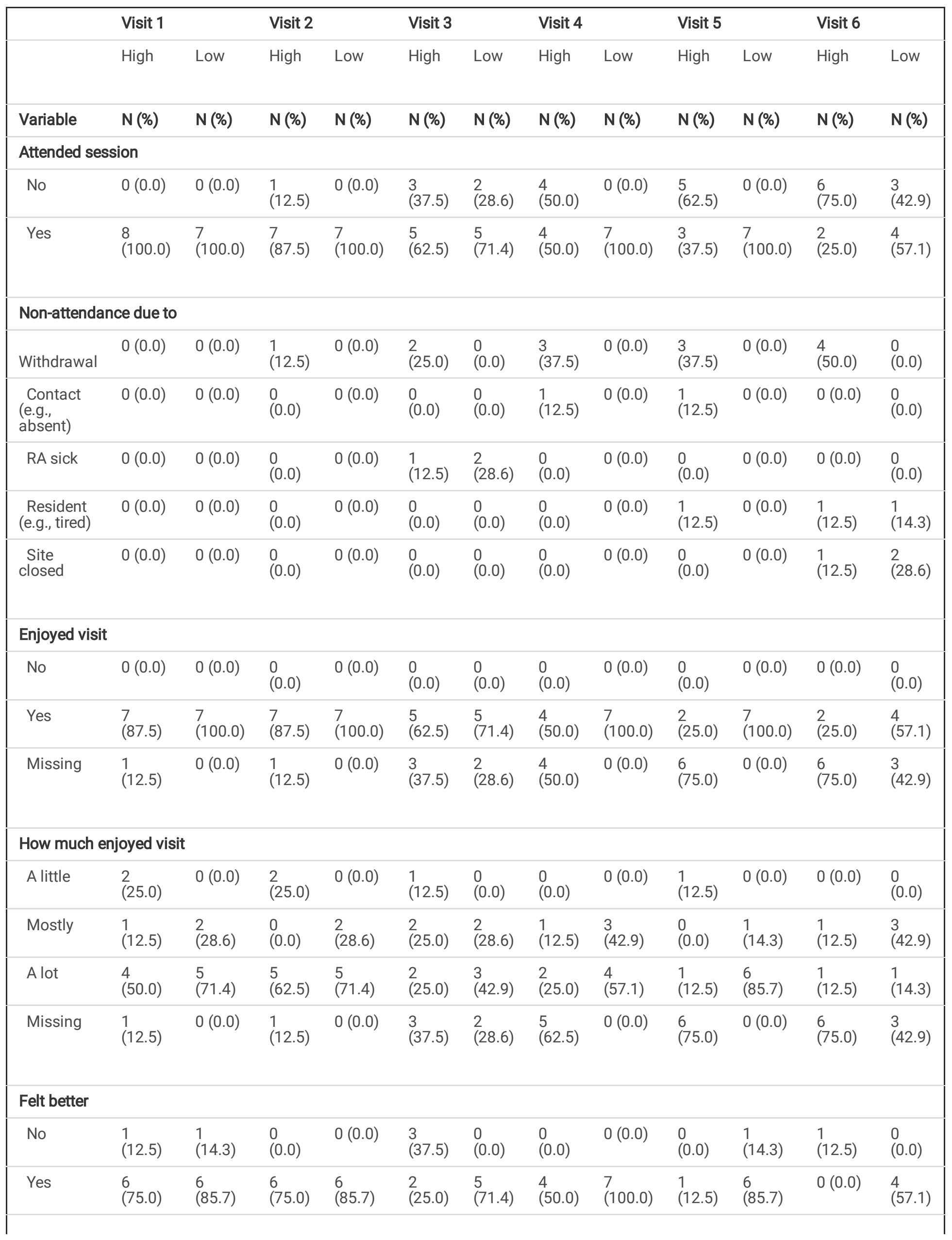

Page 19/21 


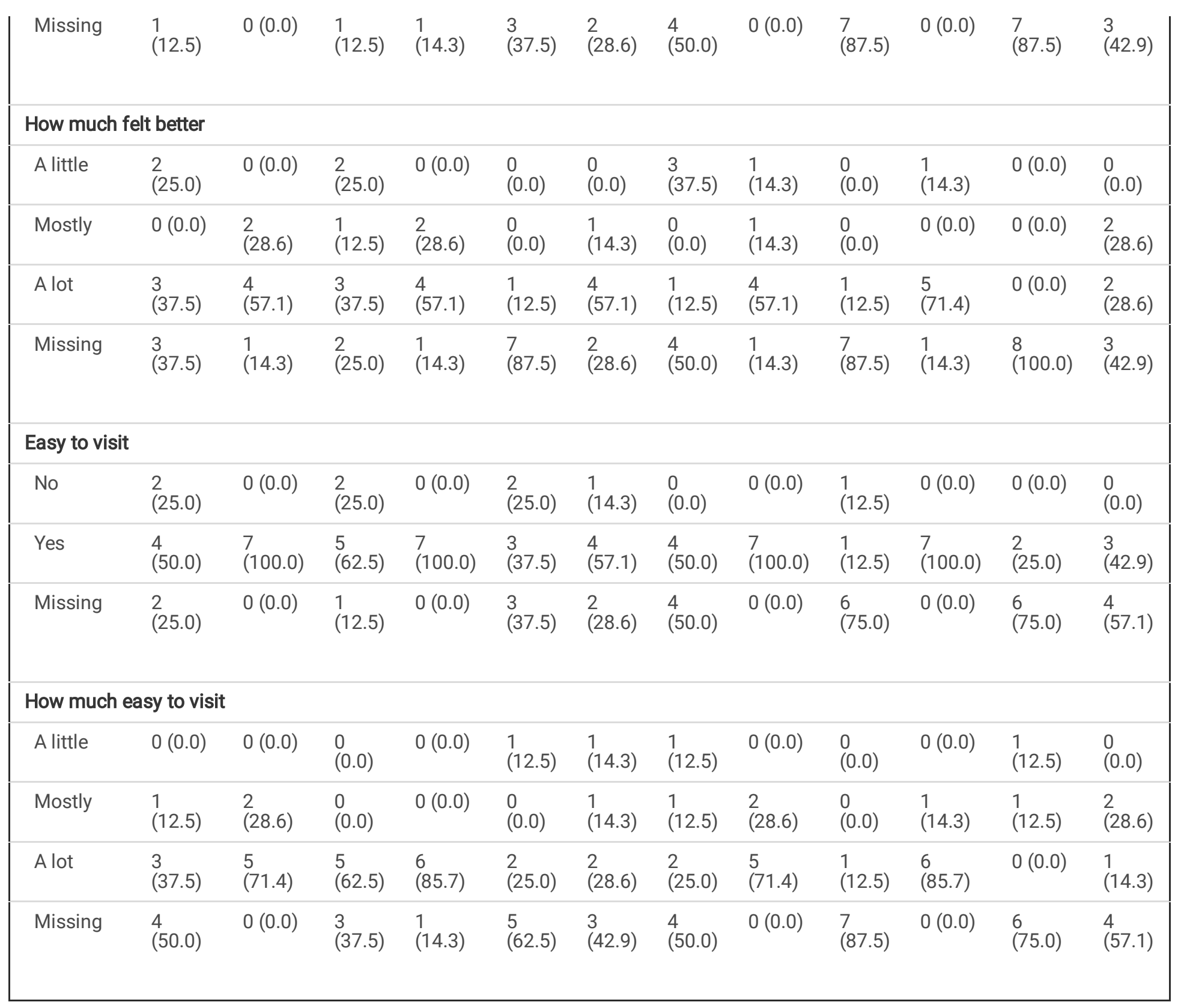

Table 3 note: Missing data includes withdrawals, non-attendance, and non-response.

Figures

Page 20/21 
Withdrawal: Resident too tired/busy ( $n=1$ )

Withdrawal: Resident passed away $(n=1)$

Missing data: RA unable to attend $(n=1)$

Withdrawal: Resident agitated by questions ( $n=1$ )

Missing data: Contact unable to attend $(n=1)$

Missing data: Contact unable to attend ( $n=1)$; Resident ill ( $n=1)$

Withdrawal: Resident moved facilities ( $n=1$ )

Missing data: Resident ill ( $n=1)$; Influenza outbreak $(n=1)$

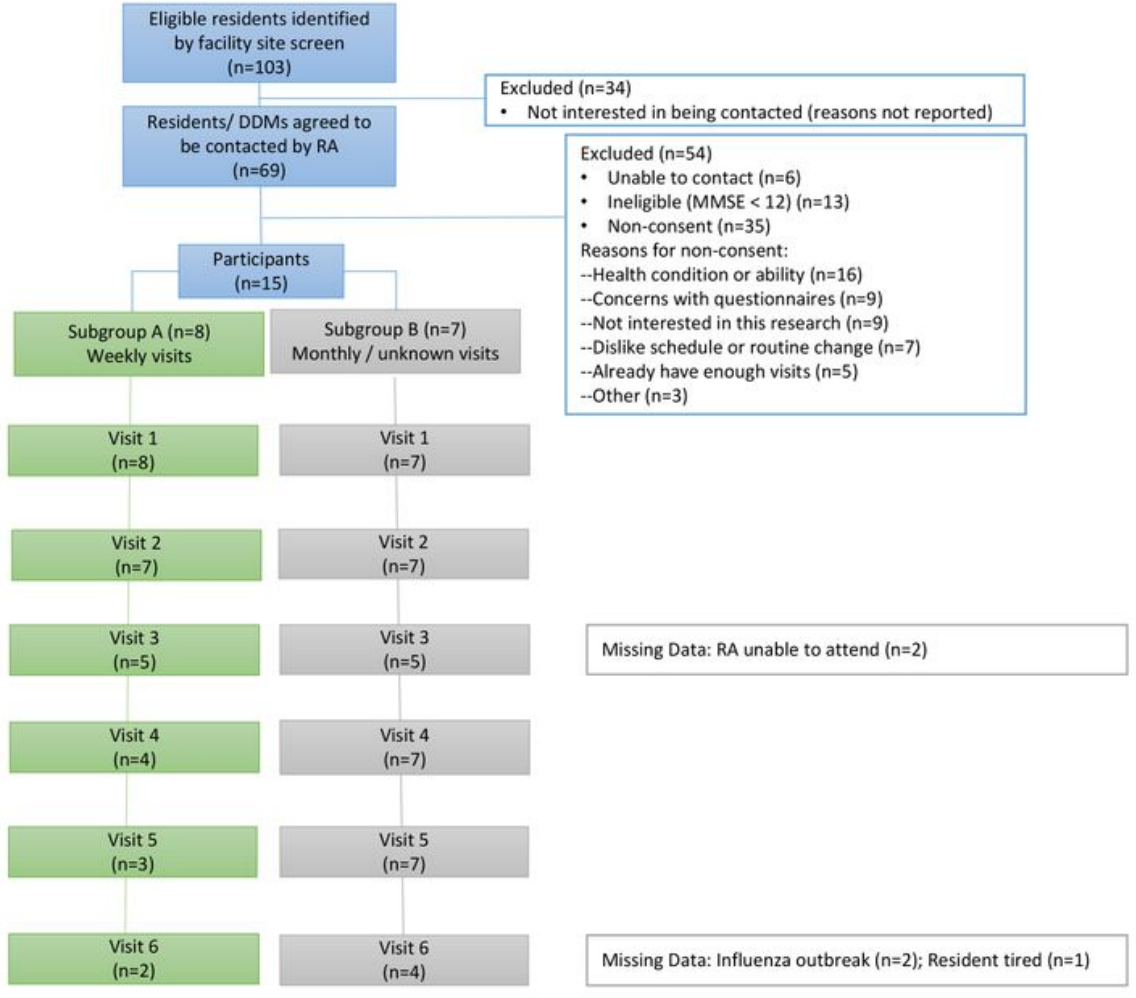

Figure 1

CONSORT flow diagram

\section{Supplementary Files}

This is a list of supplementary files associated with this preprint. Click to download.

- TitlepageAdditionalfile1.pdf

- TitlepageAdditionalfile2.pdf

- AdditionalFile1DemographicQuestionnaireresidentversion.pdf

- AdditionalFile2Acceptabilityresidentversion.pdf 